\title{
Munchausen's syndrome simulating reflex sympathetic dystrophy
}

\author{
J Rodriguez-Moreno, J M Ruiz-Martin, L Mateo-Soria, A Rozadilla, D Roig-Escofet
}

\begin{abstract}
A 15 year old girl who had pain, oedema of her left hand, and fever of four months' duration is described. Marked demineralisation of her hand was shown by radiography, and increased articular uptake by technetium-99m bone scan. All these changes were indistinguishable from reflex sympathetic dystrophy. After two admissions to hospital and multiple explorations we discovered that she had induced her symptoms herself and a diagnosis of Munchausen's syndrome was made. As far as we know this presentation has not been previously reported and might help to explain the physiopathology of some signs of reflex sympathetic dystrophy.
\end{abstract}

It is unusual to find the term Munchausen's syndrome in rheumatological reports because it is essentially a psychiatric disorder, defined by the Diagnostic and Statistical Manual of Mental Disorders as the presentation of physical symptoms that are apparently under a patient's voluntary control. ${ }^{1}$ This disorder must be distinguished from conversion disorders or malingering. Usually the patient requires multiple admissions to hospital and has the secondary effects of unnecessary explorations and treatments.

We present the first case, to our knowledge, of Munchausen's syndrome presenting as reflex sympathetic dystrophy. We have not delved into the psychopathological aspects of the syndrome, more characteristic of psychiatric reports, but this case illustrates the poorly defined limits of the reflex sympathetic dystrophy syndrome and might explain the physiopathology of some of the manifestations of this disorder, such as the regional osteoporosis or the bone scan uptake.

\section{Case report}

A 15 year old girl presented with a four month history of pain, swelling, impaired motion of the left hand, and fever. She did not remember a previous trauma. She was admitted to hospital in another centre, where multiple explorations were performed, including a biopsy of the dorsum of the wrist, without any result.

She was admitted to our department in June 1986. At the first examination the patient looked well and her axillary temperature was $38^{\circ} \mathrm{C}$. Abdominal, cardiac, respiratory, and neurological explorations showed normal results. Exploration of the locomotor apparatus showed a striking, diffuse oedema without changes in colour and temperature (fig 1), tenderness, and impaired motion of the left hand and wrist. Elbow, shoulder, and the other articular regions were normal.

Laboratory studies showed the following values: erythrocyte sedimentation rate (Westergren) $3 \mathrm{~mm} / \mathrm{h}$; packed cell volume $39 \cdot 5$; haemoglobin $127 \mathrm{~g} / \mathrm{l}$; platelet count $213 \times 10^{9} / 1$; white blood cell count $5.01 \times 10^{9} / 1$ with a normal differential count. Normal results were obtained for glucose, serum electrolytes, creatinine, calcium, phosphorus, bilirubin, serum glutamic oxaloacetic transaminase, serum glutamic oxalopyruvic transaminase, alkaline phosphatase, creatine kinase, prothrombin time, activated partial thromboplastin time, and urine analysis. Serological tests for hepatitis B antigen and antibody, antinuclear antibodies, rheumatoid factor, brucella, and lues were negative. Purified protein derivative skin test and cultures of blood and urine were also negative. Radiology of the hands showed increased juxtaarticular osteoporosis (fig 2), and a technetium$99 \mathrm{~m}\left({ }^{99 \mathrm{~m}} \mathrm{Tc}\right)$ bone scan showed marked uptake of ${ }^{99 \mathrm{~m}} \mathrm{Tc}$ in all joints of the left hand (fig 3).

Despite the normality of the laboratory tests and because of the fever, radiological and bone scan changes we performed a synovial biopsy of the wrist suspecting an infection. Examination showed only interstitial oedema without cellular infiltrates. Bacterial, mycobacterial, and fungal cultures were negative. Because of the absence of infection we started treatment of the symptoms with low doses of steroids (15 mg/day methylprednisolone) and postural treatment, with poor results. The patient was discharged.

At the next consultation it was clear that the oedema of the hand had increased and the patient and family confirmed fever; we decided to admit her again to hospital. On this second occasion no changes were seen in the physical, analytical, radiological, and bone scan examinations. Computed tomography of both wrists only showed marked demineralisation of all carpal bones without erosions.

We were puzzled and did a further biopsy of the dorsum of the wrist, but the histological findings were again normal. Cultures of surgical samples were also negative.

When we had carried out many diagnostic tests and could not think of a different approach the suspicion grew that the patient might herself have induced her symptoms. This led us to watch the patient with increased vigilance. We discovered that she had induced the 'fever' by rubbing the thermometer and produced the symptoms of the hand with a tourniquet on the wrist (see the mark in fig 1). A diagnosis of
Correspondence to: Dr Rodriguez-Moreno.

Accepted for publication 13 December 1989 


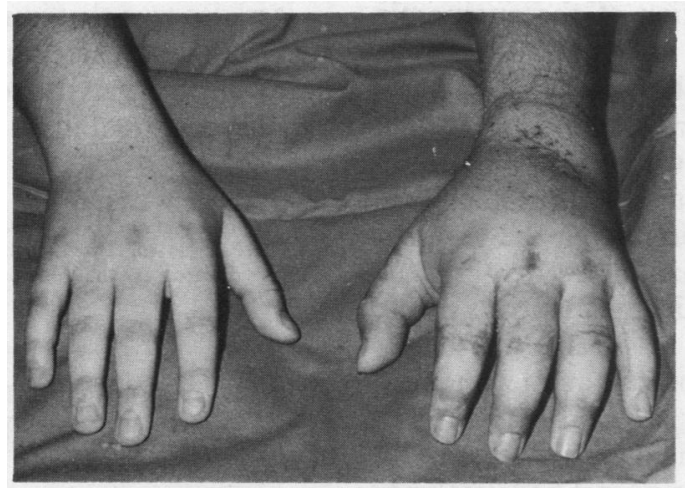

Figure 1 Diffuse oedema of the left hand. Note the hallmark of the tourniquet around the wrist.

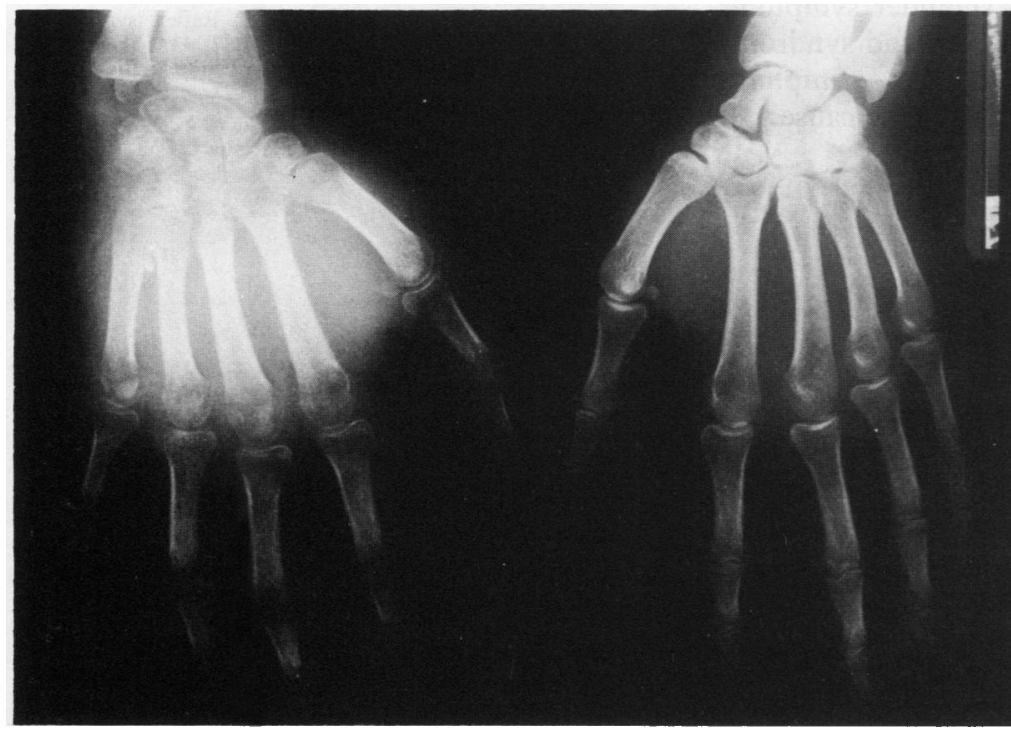

Figure 2 Striking juxta-articular osteoporosis.
Munchausen's syndrome was made, and the patient was referred to the psychiatric department.

Two months later we saw the patient again. The swelling and osteoporosis of the hand had disappeared, but now the patient simulated a contraction in adduction of the left wrist. The family was informed of the situation, but we have not seen the patient since then.

\section{Discussion}

Munchausen's syndrome is a rare psychiatric condition that has also been called hospital hoboes, peregrinating problem patients, chronic factitious illness, and hospital addicts. ${ }^{2}$ The interrelations between Munchausen's syndrome, malingering, and hysteria are incompletely understood. The proposed DMS II classification of factitious illness suggests a continuum from hysteria at one end of the spectrum to malingering at the other. ${ }^{1} \mathrm{~A}$ broad spectrum of simulated disorders related to Munchausen's syndrome has been reported: abdominal emergency, ${ }^{3}$ haemorrhagic disorders, ${ }^{4}$ neurological syndromes, ${ }^{5}$ dermatitis, hyperpyrexia, endocrine disturbances, ${ }^{6}$ and child abuse (Munchausen's syndrome by proxy). ${ }^{7}$ Practically all organs can be affected and more unusual syndromes can be found. Our case represents, to our knowledge, the first case of Munchausen's syndrome mimicking a reflex sympathetic dystrophy syndrome.

Reflex sympathetic dystrophy is a clinical syndrome of unknown origin characterised by pain, oedema, vasomotor disturbances, regional osteoporosis, and juxta-articular uptake in ${ }^{99 \mathrm{~m}} \mathrm{Tc}$ bone scan. Although the exact cause of this syndrome remains unclear, most authors accept
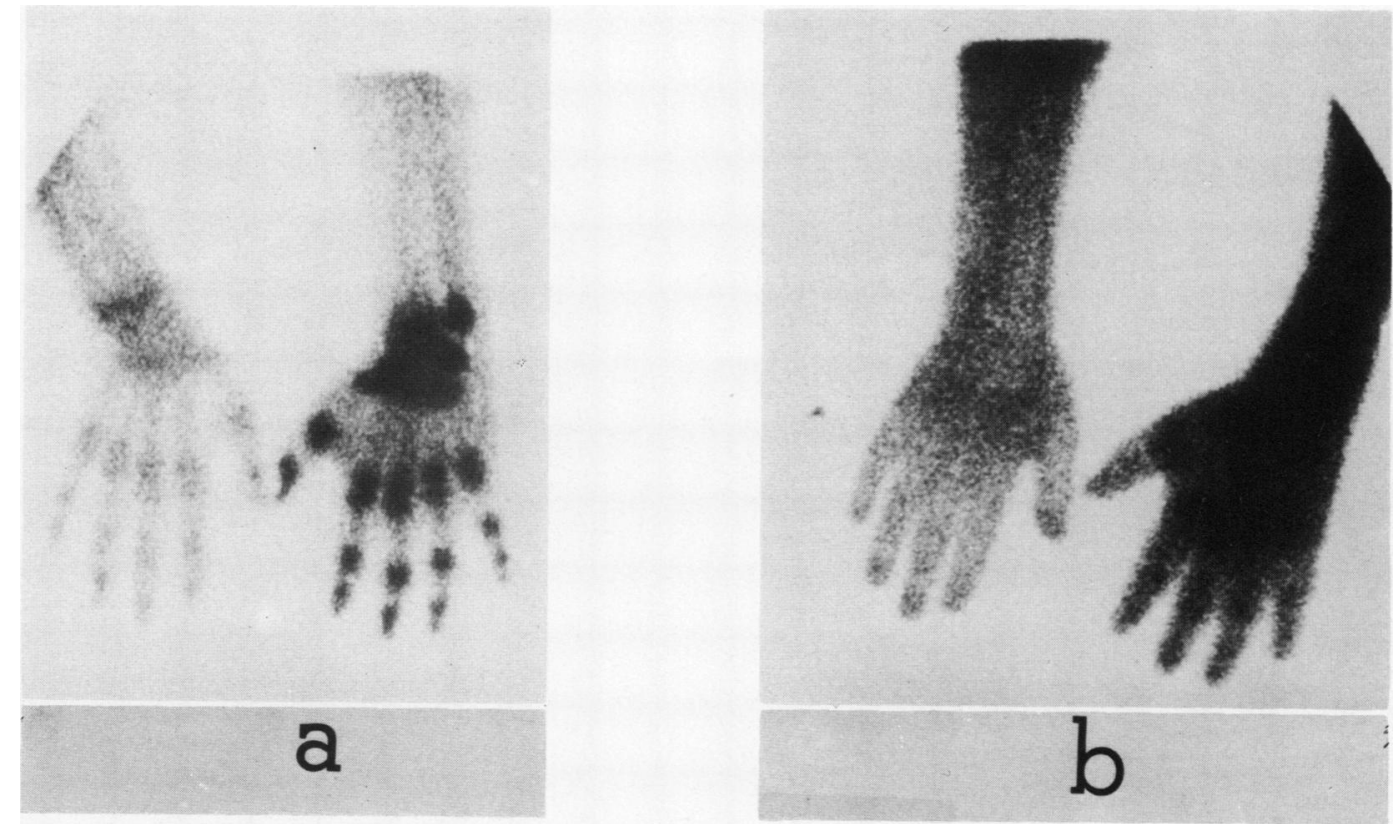

Figure 3 (a) Early bone scan with technetium-99m $\left({ }^{99 m} T c\right)$. (b) Late bone scan with ${ }^{99 m} T c$. Note the increased uptake of technetium by all joints of the left hand. 
that there is a disregulation of the autonomic nervous system. ${ }^{8}$

Some authors have shown a blood stasis secondary to vasomotor changes in reflex sympathetic dystrophy using early dynamic bone scans. ${ }^{9}$ Might this stasis induce metabolic changes in bones, thus producing the regional osteoporosis? This remains to be proved. Clearly, a tourniquet applied to a limb, as in our case, can induce venous stasis and this might induce the regional osteoporosis and technetium uptake on bone scan. For ethical reasons it was not possible to confirm this hypothesis by reproducing the syndrome after it had resolved.

It is possible that the compression of the tourniquet might induce a true reflex sympathetic dystrophy syndrome. This is unlikely in our case because the vasomotor and other 'sympathetic' changes and shoulder symptoms usually present in the shoulder-hand syndrome were absent. Additionally, the symptoms resolved rapidly after removal of the cause.
It is also true that immobilisation can produce juxta-articular osteoporosis and abnormal bone scan, but we may disregard this as the patient was never immobilised with splints.

1 Diagnostic and statistical manual of mental disorders. Washington DC: American Psychiatric Association, 1980: 331-2.

2 Case records of the Massachusetts General Hospital (case 28-1984). N Engl f Med 1984; 311: 108-15.

3 Justus P G, Kreutziger S S, Kitchens C S. Probing the dynamics of Munchausen's syndrome. Ann Intern Med 1980; 93: 120-7.

4 Feinsilver S J, Raffin J A, Kornei M C, Sullivan S J, Smith M A. Factitious hemoptysis. The case of the red towel. Arch Intern Med 1983; 143: 567-8.

5 Batshaw M L, Wachtel R C, Deckel A W, et al. Munchausen's syndrome simulating torsion dystonia. $N$ Engl $₹$ Med 1985; 312: 1437-9.

6 Witt M E, Ginsberg-Fellner F. Prednisone-induced Munchausen syndrome. Am $f$ Dis Child 1981; 135: 852-3.

7 Amegavie L, Marzouk O, Mullen J, Sills J, Gauthier Le Tendre J. Munchausen's syndrome by proxy: a warning for health professionals. Br Med F 1986; 293: 855-6.

8 Schwartzman R J, McLellan T L. Reflex sympathetic dystrophy. A review. Arch Neurol 1987; 44: 555-61.

9 Gaucher A, Raul P, Wiederkhr P, et al. Etude scintigraphique des algodystrophies réflexes. Rev Rhum Mal Osteoartic 1982; 49: 841-6. 